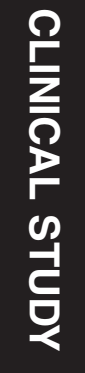

${ }^{1}$ Manchester Centre for Health Economics, The University of Manchester, Manchester, UK

${ }^{2}$ Manchester Centre for Genomic Medicine, Institute of Human Development, Faculty of Medical and Human Sciences, University of Manchester, Manchester Academic Health Science Centre (MAHSC), Saint Mary's Hospital, Oxford Road, Manchester, UK

${ }^{3}$ Manchester Centre for Genomic Medicine, Central Manchester University Hospitals NHS Foundation Trust, MAHSC, Saint Mary's Hospital, Oxford Road, Manchester, UK

${ }^{4}$ Centre for Medical Education, School of Medicine, Cardiff University, Cardiff, UK

Correspondence:

G Black, Manchester Centre for Genomic Medicine, Central Manchester University Hospitals NHS Foundation Trust, Manchester Academic Health Sciences Centre (MAHSC), St Mary's Hospital, Oxford Road, Manchester M13 9WL, UK Tel: +44 (0)161276 6269; Fax: +44 (0)1612766145. E-mail: graeme.black@ manchester.ac.uk

Received: 15 December 2015 Accepted in revised form: 15 February 2016 Published online: 15 April 2016

\section{Identifying variation in models of care for the genomic-based diagnosis of inherited retinal dystrophies in the United Kingdom}

\author{
Abstract \\ Purpose Advances in genomic technologies \\ are prompting a realignment of diagnostic \\ and management pathways for rare inherited \\ disease. New models of care are being \\ developed as genomic-based diagnostic \\ testing becomes increasingly relevant within \\ more and more aspects of medicine. This \\ study describes current care models for the \\ provision of a genomic-based diagnosis for \\ patients with inherited retinal dystrophy \\ (IRD) in UK clinical practice. \\ Methods A structured telephone survey, \\ conducted (in 2014) with all 23 UK Regional \\ Genetics Centres and a sample of specialist \\ ophthalmology centres $(n=4)$, was used to \\ describe models of service delivery and \\ current levels of genomic-based diagnostic \\ testing. Quantitative data were summarised \\ using descriptive statistics. Responses to \\ open-ended questions were summarised \\ using thematic analysis. \\ Results Of the 27 centres 10 of them saw \\ IRD patients in 'generic' clinics and $\mathbf{1 7}$ \\ centres offered ophthalmic-specific clinics. \\ Extensive regional variation was observed in \\ numbers of patients seen and in how care for \\ the diagnosis and management of IRD was \\ provided. \\ Conclusions Understanding current practice \\ is a necessary first step in the development \\ and evaluation of complex interventions, such \\ as care models for the genomic-based \\ diagnosis of inherited eye conditions. \\ Presented findings here relating to disparities \\ in care provision are potentially linked to \\ previously reported evidence of perceived \\ unmet needs and expectations of IRD service \\ users. This work provides a foundation for \\ the integration of new care models in \\ mainstream medicine.
}

M Eden ${ }^{1}$, K Payne ${ }^{1}$, C Jones $^{1}$, SJ Wright ${ }^{1}$, G Hall ${ }^{2,3}, \mathrm{M} \mathrm{McAllister}^{4}$ and $\mathrm{G}$ Black $^{2,3}$
Eye (2016) 30, 966-971; doi:10.1038/eye.2016.74; published online 15 April 2016

\section{Introduction}

Inherited retinal dystrophies (IRD) are a group of genetically and clinically heterogeneous inherited eye conditions that result in vision loss. For the majority of patients there is no effective preventative treatment, although novel therapies, are in development. ${ }^{1}$ Consequently, the present focus is upon accurate diagnosis and conservative management, aligned to genetic counselling and molecular testing. ${ }^{2-4}$ Despite individual components of care for IRD demonstrating clinical utility, the delivery of models of care in clinical settings in the United Kingdom has been reported to fall below services users' needs and expectations. ${ }^{5}$

A 2008 report identified significant variation in the extent of service delivery for IRD across the UK. ${ }^{6}$ Recent studies have underlined that, while service users and providers concur about which elements of care are desirable, no precise care model of best practice exists. ${ }^{3,5}$ The body of evidence indicating regional inequities has informed a recent NHS England policy directive to re-organise specialist ophthalmology services under agreed national guidelines. ${ }^{7}$ To facilitate this re-organisation, new care models that can be implemented in a consistent way across the health service are required.

Recognition of the need for the standardisation of IRD services comes at a time of unprecedented change as next-generation sequencing (NGS) is becoming integrated into mainstream practice, ${ }^{8}$ increasing the opportunities for patients to derive clinical utility from genetic tests. ${ }^{9}$ To ensure that the introduction of such new technologies improve, rather than compound, 
problems of regional variation, it is necessary to equip clinicians and service users to develop standardised care models and clear guidance on best practice. Since care models for IRD incorporate multiple components, for example, counselling, genomic testing, and clinical care, they are best viewed as a 'complex intervention'. The Medical Research Council guidance ${ }^{10}$ on the development and evaluation of complex interventions outlines a need to understand current practice in order to inform the design of future interventions and to establish whether new working practices offer improvements at an acceptable cost. The aim of this paper is to describe current care models for IRD in the UK, so that as novel care models are developed, knowledge of current practice can be used to determine their relative effectiveness and value-for-money.

\section{Materials and methods}

A UK-wide semi-structured telephone survey was used to describe current care models for IRD.

\section{Sampling frame}

Healthcare professionals from Regional Genetics Centres (RGCs) and specialist ophthalmology centres in the United Kingdom who provide IRD in secondary care settings were recruited through existing formal and informal networks. RGCs were identified from the British Society for Genomic Medicine's website. ${ }^{11}$ To our best knowledge these are the centres who have a significant role in UK care provision for IRD. At the time of the survey, we asked each respondent if they knew of other centres providing IRD care in their region. We ensured that there was no duplication of responses; each ophthalmology centre was distinct from any RGC. Taken as a whole, therefore, they provide a comprehensive overview of IRD care provision in the UK-geographical coverage is supplied in the Supplementary Material.

\section{Survey design}

A literature review was undertaken to inform the survey structure, which was refined using interdisciplinary group meetings of five experts (two health economists; two genetic counsellors; and a consultant in ophthalmic genetics). The telephone survey was piloted $(n=3)$ and minor changes to questions made. The final survey comprised a structured schedule of open and closed questions about the scope and scale of clinic workload and key elements of care (Supplementary Appendix 1).

\section{Data collection}

One researcher (ME) collected responses to the survey using telephone interviews. The answers to closed questions were recorded in a bespoke database. Interviews were audio-recorded to allow transcription of responses to open-ended questions and these data were supplemented using additional field notes.

\section{Data analysis}

Having identified all relevant providers of IRD care, survey data were analysed to describe and compare current care provision. Descriptive statistics were used to summarise quantitative data. Data from open-ended questions were collated and analysed using thematic analysis. $^{12}$

\section{Results}

\section{Study sample and structure of services}

Every RGS in the United Kingdom $(n=23)$ and four specialist ophthalmology centres offering IRD services were represented (Supplementary Appendix 2).

Respondents comprised 20 medically trained consultants, six Genetic Counsellors and one Specialist Registrar all directly involved in the provision of care for IRD.

Ten respondents (37\%) provided 'generic' clinics, within clinical genetics departments, for patients with IRD to be seen outside of their normal ophthalmic care. The remit of these 'generic' clinics extended beyond IRD to include a broad range of inherited conditions (hereafter labelled generic). Twelve respondents (44\%) described 'dedicated' ophthalmic genetic clinics exclusively provided for patients affected by inherited eye conditions (hereafter labelled dedicated). Five respondents (19\%) described providing both generic and dedicated clinics (hereafter labelled dedicated). There was no statically significant difference (Mann-Whitney test; $P=0.158)$ in the median duration of dedicated $(48.75 \mathrm{~min})$ and generic ophthalmology (41.25 min) clinic appointments.

All centres providing generic clinics had input from a consultant geneticist at the initial clinic appointment although one of these centres reported use of that a separate, preceding genetic counsellor-led appointment. Genetic counsellors were involved in 4 of the10 generic clinics and 12 of the 17 dedicated ophthalmology clinics. Involvement of a consultant ophthalmologist in the initial appointment was reported by only one centre providing a generic clinic compared with 14 of the 17 $(82 \%)$ centres running dedicated ophthalmic genetic clinics. Only one respondent representing a centre providing IRD care in generic clinics reported being 
able to access support from optometry and vision scientists during the clinic appointment. However, reported availability of optometry support was high in centres who provided dedicated ophthalmology clinics with over four-fifths of these centres ( $n=14 / 17 ; 82 \%)$ being able to involve optometrists in their clinics.

There was reported variation in access to an Eye Clinic Liaison Officer (ECLO) $(n=6 / 27 ; 22 \%)$ and none of centres providing generic clinics had access to an ECLO. Five respondents $(n=5 / 27 ; 19 \%)$ stated that their centres have direct access to social workers. All respondents with one exception $(n=26 / 27 ; 96 \%)$ reported that their centre had access to translation services. Clinic and administrative support was typically provided by administrators employed to support their centre's broader needs rather than having a specific remit to organise services for IRD.

\section{Caseloads}

Respondents were asked if they saw patients with IRD/ retinitis pigmentosa as well as with other ophthalmic genetic conditions including childhood glaucoma, optic neuropathy or congenital cataracts. All reported that their remit included providing services for patients and their families with a wide range of IRD and referrals for a wide range of ophthalmic genetic conditions were reported by the majority $(n=26 / 27 ; 96 \%)$ of respondents. Typically, service providers reported seeing 10 to 20 patients every month; $41 \%(n=11 / 27)$ of respondents reported seeing this volume of patients regularly. With the exception of two RGCs, caseloads comprised both children and adult patients. In eight $(n=8 / 27 ; 30 \%)$ clinics caseloads predominately comprised children while the other centres reported that adult patients formed the bulk of their caseload.

Differences in reported monthly caseloads (Figure 1) were observed between centres providing IRD care within generic clinics and those providing dedicated clinics (4.5 compared with 18.1 patients per month, respectively). No centre providing generic clinics reported seeing more than ten IRD patients per month compared with almost half $(n=8 / 17 ; 47 \%)$ of those offering dedicated ophthalmic genetics clinics.

\section{Referral patterns}

The majority of referrals ( $n=19 / 27 ; 70 \%$ ) came from hospital ophthalmology departments although referrals from paediatric departments were also common ( $n=24 / 27 ; 89 \%)$. All but one centre received referrals directly from primary care. Direct-from-optician referrals were less common with a third of respondents $(n=9 / 27$; $33 \%$ ) reporting this referral route, eight of whom were dedicated clinics. All respondents from centres providing only generic clinics for IRD $(n=10 / 10 ; 100 \%)$ reported ophthalmology to be the main source of referrals. For centres providing dedicated ophthalmic genetic clinics, ophthalmology was cited as the main source of referrals by 12 centres $(n=12 / 27 ; 71 \%)$ with the remainder of centres $(n=5 / 17 ; 29 \%)$ reporting that a main source of referral was GP/primary care.

\section{Nature of services provided}

Responses to an open-ended question were analysed using themes to describe the nature of the care models for IRD. This analysis demonstrated that after a detailed history, examination plus ophthalmic imaging (in dedicated ophthalmology clinics), diagnoses and prognoses are shared with patients during initial appointments and the relevant management options discussed. Practical help around vision loss, which can include help with registration and signposting to relevant services, was frequently also provided. During the consultations, the potential role of genetic testing is discussed and, where appropriate, a blood sample is taken.

In terms of the management or treatment options provided, over half of the respondents from participating centres providing generic clinics $(n=6 / 10 ; 60 \%)$ reported that a referral back to ophthalmology was often the only course of action. Two respondents from centres providing generic clinics said that no treatment or management was offered. One respondent reported pre-natal diagnosis as a treatment/management option and two said that they would seek entry for their patients into clinical trials if appropriate.

Genetic testing was reported to be available for IRD patients in all centres. Each respondent was asked whether their centre had ever requested a genetic test for a range of IRDs. No discernible difference in test request activity was observed between centres who offered dedicated compared with genetic clinics. Table 1 shows that a high proportion of centres had experience of ordering tests for each condition.

The primary reasons for ordering genetic tests were patient-driven and focused upon: pre-natal testing; informing reproductive choices; confirming diagnoses and for research purposes. The increasing availability of NGS tests in recent years was noted by respondents, and there was anticipation that requests for new tests were becoming more commonplace.

In all centres a summary letter dictated by the consultant or genetic counsellor would be sent to clinic attendees and the health professional who referred them. Eight ( $n=8 / 27 ; 30 \%$ ) respondents-four each from dedicated and generic clinics—estimated a single visit 


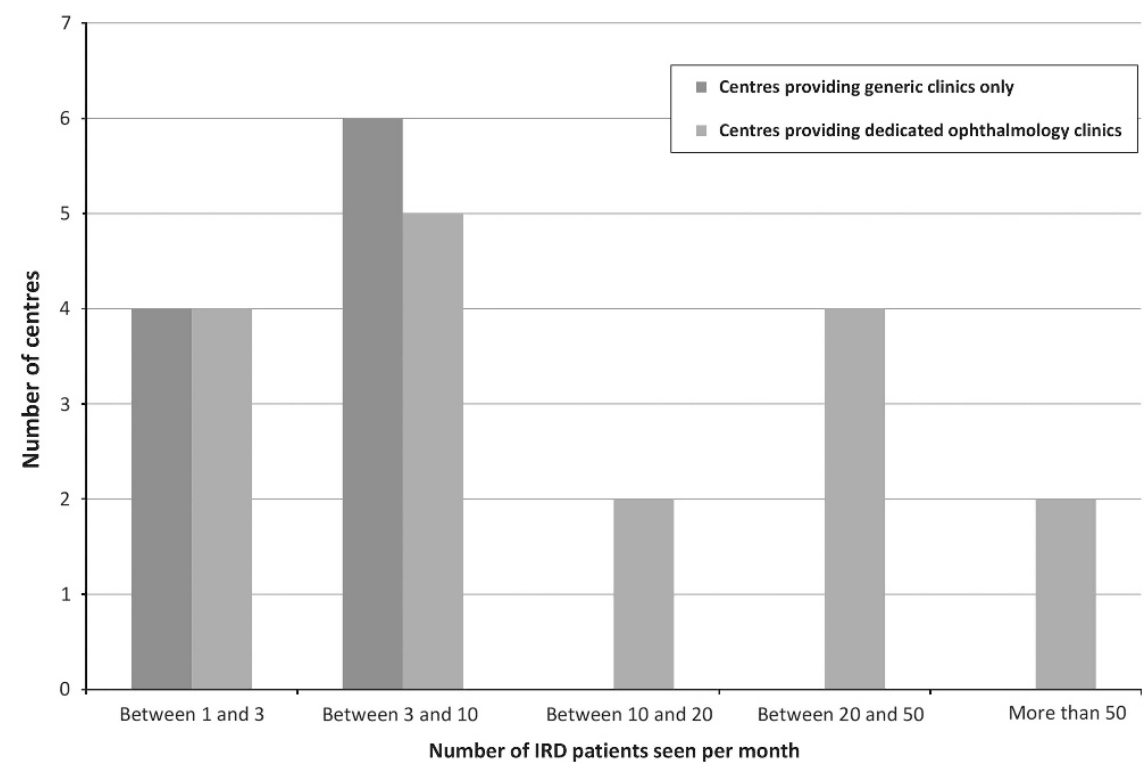

Figure 1 Caseload by clinic type provided.

Table 1 Test request activity by IRD type

\begin{tabular}{lc}
\hline RD type & $\begin{array}{c}\text { Number of respondents who reported } \\
\text { having ever requested tests }(\mathrm{n}=27 ; \%)\end{array}$ \\
\hline $\begin{array}{l}\text { Autosomal dominant } \\
\text { retinitis pigmentosa }\end{array}$ & $22(81)$ \\
$\begin{array}{l}\text { Autosomal recessive } \\
\text { retinitis pigmentosa }\end{array}$ & $19(70)$ \\
$\begin{array}{l}\text { X-linked retinitis } \\
\text { pigmentosa }\end{array}$ & $24(89)$ \\
$\begin{array}{l}\text { Other isolated IRD } \\
\text { Leber congenital } \\
\text { amaurosis }\end{array}$ & $21(78)$ \\
$\begin{array}{l}\text { Macular dystrophy } \\
\text { Bardet-Biedl syndrome }\end{array}$ & $20(74)$ \\
Usher's Syndrome & $19(70)$ \\
\hline
\end{tabular}

to be typical, five $(n=5 / 27 ; 19 \%)$ respondents (one from a dedicated clinic along with four from generic clinics) considered two visits to be usual and one respondent from a dedicated clinic (4\%) reported that three clinic attendances could be expected. The remaining 13 (44\%) respondents (eleven dedicated and two generic) did not specify the typical visit frequency. Most centres ( $n=25 / 27 ; 93 \%$ ) provided follow-up in clinic with unresolved diagnoses, syndromic implications, and the conveyance of test results being important motivators to offering follow-up.

\section{Discussion}

This study expands upon, and updates, the information available from previous studies that have sought to understand care provision for inherited eye conditions. ${ }^{6}$
A particular strength of this survey-based study is that all UK RGCs contributed and provided quantitative data that enabled a clear description of the services that are currently provided for patients with IRD. These data were also supplemented by four ophthalmology clinics. This work directly addresses questions that have arisen from multiple UK policy directives $6,7,13$ in the context of the rapidly shifting landscape of genomic medicine. Such rapid change underlines the need for a broader programme of work aimed at the standardisation of care and development of evidence-based guidelines.

Variation in clinical practice throughout the United Kingdom has been confirmed through this work; the survey data demonstrated a marked difference in the quantity of IRD patients seen across the RGCs and ophthalmology centres. Those centres providing dedicated combined genetic ophthalmology clinics-with additional staffing resources-are, on average, seeing four times more patients per month than the centres seeing IRD patients in generic clinical genetics clinics. Future research could be aimed at understanding why there is disparity in caseloads, the impact of this disparity, and the identification of potential barriers to accessing services. It may be the case, for example, that decreased levels of demand are associated with models of IRD care provided in generic clinics.

Our survey forms part of a broader programme of research seeking to improve IRD care. Previous qualitative work within this programme of work has revealed that the needs of families accessing IRD care have not always been satisfied. ${ }^{5}$ Combs et $a l^{5}$ identified how three broad categories of service user expectations could be used with which to align IRD services: families 
want (1) to obtain a diagnosis and information about risks, prognoses, and research; (2) support to enable psychological adjustment to living with IRD; and (3) information about welfare and accessing practical support. Improving access to additional resources, for example, on-site optometry services and ECLO support has the potential to address these unmet needs and expectations. For example, the need for help in identifying additional information and practical support in terms of financial issues, means of adapting to vision loss, and help with mobility could be directly addressed with care input from an ECLO.

We also recently reported evidence that there are regional inequalities in access to genetic tests for IRD. ${ }^{14}$ It is important to consider that observations from our epidemiological study and evidence of variation in care provision from this service evaluation may be linked. In our survey we did not ask about volume of test requests because we were primarily interested in understanding current care pathways (ie, whether or not a patient could expect to be offered a test) for IRDs. The epidemiological data does support the findings of our survey; in that at least some level of testing for IRD is seen throughout the UK, but the observed regional variation in testing rates is unsurprising given the current disparate nature of IRD care. It follows that there is the likelihood that any increase in referrals seen as new NGS genetic tests become available will exacerbate this situation. At a time when genomic technology is demonstrating its increasing potential to be a component of complex interventions, such work represents a first step in ensuring optimal integration into mainstream medical specialties.

\section{Summary}

What was known before

- Needs and expectations of people accessing services for inherited retinal dystrophy are often not met.

- New models of care are being developed as genomic technology continues to present further opportunities for more people to benefit from genetic test information.

- There is a need to understand how services are currently delivered so that new care models can be evaluated.

What this study adds

- All 27 UK centres have described the nature and extent of their service provision for inherited retinal dystrophy.

- Extensive regional variation was observed in numbers of patients seen and in how care is provided.

- This work will allow new care models to be evaluated in terms of their effectiveness and value-for-money.

\section{Conflict of interest}

The authors declare no conflict of interest.

\section{Author contributions}

ME collected and analysed data and produced the first draft of the article. KP, ME, GH, MM, and GCMB designed the study. CJ and SJW collated and prepared the survey for analysis. All authors analysed data and have approved the final article.

\section{Acknowledgements}

This research was funded by Fight for Sight grant number 1801 and facilitated by the Manchester Biomedical Research Centre and the Greater Manchester Comprehensive Local Research Network. The authors would like to thank survey respondents for their participation.

\section{References}

1 Jacobson SG, Cideciyan AV. Treatment possibilities for retinitis pigmentosa. $N$ Engl J Med 2010; 363(17): 1669-1671.

2 National Society of Genetic Counselors' Definition Task, Force, Resta R, Biesecker BB, Bennett RL, Blum S, Hahn SE et al. A new definition of Genetic Counseling: National Society of Genetic Counselors' Task Force report. J Genet Couns 2006; 15(2): 77-83.

3 Combs R, McAllister M, Payne K, Lowndes J, Devery S, Webster AR et al. Understanding the impact of genetic testing for inherited retinal dystrophy. Eur J Hum Genet 2013; 21(11): 1209-1213.

4 Eden M, Payne K, Combs RM, Hall G, McAllister M, Black GCM. Valuing the benefits of genetic testing for retinitis pigmentosa: a pilot application of the contingent valuation method. Br J Ophthalmol 2013; 97(8): 1051-1056.

5 Combs R, Hall G, Payne K, Lowndes J, Devery S, Downes SM et al. Understanding the expectations of patients with inherited retinal dystrophies. Br J Ophthalmol 2013; 97(8): 1057-1061.

6 Moore T, Burton H. PHG Foundation I Genetic ophthalmology in focus: a needs assessment and review of specialist services for genetic eye disorders. Available at http://www.phgfoundation.org/reports/4984/ (Accessed on 13 February 2014).

7 Anon. NHS England» D12. Specialised Ophthalmology Services. Available at https://www.england.nhs.uk/ commissioning/spec-services/npc-crg/group-d/d12/ (accessed on 13 February 2014).

8 Gillespie RL, Hall G, Black GC. Genetic testing for inherited ocular disease: delivering on the promise at last? Clin Experiment Ophthalmol 2014; 42(1): 65-77.

9 O'Sullivan J, Mullaney BG, Bhaskar SS, Dickerson JE, Hall G, O'Grady A et al. A paradigm shift in the delivery of services for diagnosis of inherited retinal disease. J Med Genet 2012; 49(5): 322-326.

10 Craig P, Dieppe P, Macintyre S, Michie S, Nazareth I, Petticrew M. Developing and evaluating complex interventions: the new Medical Research Council guidance. BMJ 2008; 337: a1655.

11 Anon. British Society for Genetic Medicine. Available at http:/ / www.bsgm.org.uk/ (accessed on 3 July 2014).

12 Braun V, Clarke V. Using thematic analysis in psychology. Qual Res Psychol 2006; 3(2): 77-101. 
13 Anon. Genomic Technology in Healthcare: Building On Our Inheritance-Publications-GOV.UK. Available at https://www.gov.uk/government/publications/genomictechnology-in-healthcare-building-on-our-inheritance (accessed on 13 February 2014).
14 Harrison M, Birch S, Eden M, Ramsden S, Farragher T, Payne $\mathrm{K}$ et al. Variation in healthcare services for specialist genetic testing and implications for planning genetic services: the example of inherited retinal dystrophy in the English NHS. J Community Genet 2015; 6(2): 157-165.

Supplementary Information accompanies this paper on Eye website (http://www.nature.com/eye) 